\title{
Loss aversion at the aggregate level across countries and its relation to economic fundamentals ${ }^{\text {th }}$
}

\author{
Reto Foellmi ${ }^{\mathrm{a}}$, Adrian Jaeggi ${ }^{\mathrm{a}}$, Rina Rosenblatt-Wisch*,b \\ ${ }^{a}$ University of St.Gallen, Department of Economics, SIAW-HSG, Bodanstrasse 8, St.Gallen CH-9000, Switzerland \\ ${ }^{\mathrm{b}}$ Swiss National Bank, Börsenstrasse 15, Zürich CH-8022, Switzerland
}

\section{A R T I C L E I N F O}

\section{JEL classification:}

E21

O41

Preferences

Loss aversion

Prospect theory

GMM

\begin{abstract}
A B S T R A C T
Preferences are important when thinking about macroeconomic problems and questions. Differences in preferences might, for example, explain cross-country variations in economic fundamentals.

In recent years, differences in preferences across countries and cultures have been studied more frequently, usually concentrating on micro evidence. However, it is an open question as to how differences in average preferences affect the aggregate economy. Coming from a macroeconomic perspective, we test whether preferences stated in Kahneman and Tversky's prospect theory, namely, reference point dependence and loss aversion, prevail on the aggregate and whether the average degree of loss aversion differs across countries.

We find evidence of loss aversion for a broad set of OECD countries, while the average loss aversion clearly differs across these countries. We find little evidence that these differences could be linked to micro evidence. Furthermore, we analyse whether the different degrees of loss aversion correlate with economic fundamentals such as the level of GDP and consumption per capita. We find that indeed loss aversion is negatively correlated with GDP and consumption per capita and positively correlated with consumption smoothing.
\end{abstract}

\section{Introduction}

Preferences are important features in macroeconomic modelling. Differences in preferences might correlate with aggregate economic fundamentals. In recent years, differences in preferences across countries and cultures have been studied more frequently. Several papers found differences in preferences across cultures and/ or countries using evidence generated at the micro level, in the form of surveys or experiments (see e.g. Rieger et al., 2015; Herrmann et al., 2008; Vieider et al., 2015).

To gain progress in determining whether differences in preferences matter for aggregate outcomes, our paper approaches this from the opposite direction: We start from a purely macroeconomic perspective and test whether preferences, namely, reference point dependence and loss aversion, two key elements of Kahneman and Tversky's prospect theory, vary across countries by only using a macroeconomic time series. To do so, we follow Rosenblatt-Wisch (2008), in which she introduced prospect theory in a stochastic version of the Ramsey-Cass-Koopmans optimal growth model. The preferences of the representative agent in that model are given by the experimentally validated prospect utility function of Kahneman and Tversky (1979) and Tversky and Kahneman (1992). She then

\footnotetext{
We would like to thank seminar participants at the Swiss National Bank and at the University of St.Gallen for their helpful comments. The views expressed in this paper are those of the authors and do not necessarily represent those of the Swiss National Bank. Declarations of interest: none.

* Corresponding author.

E-mail address: rina.rosenblatt@snb.ch (R. Rosenblatt-Wisch).
} 
tested the model with US data and found evidence of loss aversion in a US macroeconomic time series, in line with the values found by Kahneman and Tversky (1979) and Tversky and Kahneman (1992).

Our contribution is two-fold. First, we test empirically for loss aversion across countries for the aggregate economy. We find that loss aversion prevails at the aggregate level in all countries and that the average degree of loss aversion clearly differs across countries. To check whether these degrees of loss aversion could be linked to micro data, we apply the cultural dimensions constructed by Hofstede et al. (2010) and data from the World Values Survey. Because of the large heterogeneity of the data, we find little statistical evidence that either the Hofstede dimensions or the World Values Survey data match with the cross-country variations in the estimated loss aversion.

Second, we analyse whether the different degrees of loss aversion correlate with economic fundamentals such as GDP and consumption per capita. We find that indeed, according to our analysis, loss aversion is negatively correlated with GDP and consumption per capita and is positively correlated with consumption smoothing. These empirical results are in line with the theoretical ones found by Foellmi et al. (2011).

We concentrate on two key elements of Kahneman and Tversky's experimentally validated prospect theory, namely, reference point dependence and loss aversion. In a recent survey on thirty years of prospect theory, Barberis (2013) notes that the concept of loss aversion relative to a reference point could be promising when thinking about macroeconomics. Focusing on these two aspects of prospect theory, namely, reference point dependence and loss aversion, is common for analysing the aggregate level. Barberis et al. (2001) apply these aspects in order to assess the aggregate stock market behaviour, and Benartzi and Thaler (1995) study the equity premium under loss aversion. The paper by Berkelaar et al. (2004) uses GMM to estimate loss aversion in the aggregate U.S. stock market. They find an implied loss aversion coefficient of the same size as the one found by Tversky and Kahneman (1992). Kahneman and Tversky (1979) formulated their theory on individual choice under uncertainty. The above-cited papers find loss aversion even in aggregate market data. Brooks and Zank (2005), Köszegi and Rabin (2006) and Abdellaoui et al. (2007) found experimental evidence of loss aversion at the aggregate level. In addition, loss aversion and thinking in differences have also been found in purely deterministic models (see e.g. Thaler, 1980; Kahneman et al., 1990; Tversky and Kahneman, 1991). Chen et al. (2006) find, in an experiment with Capuchin monkeys, that these two behavioural biases even extend beyond species and may be innate, rather than learned.

The rest of the paper is structured as follows. Section 2 reviews the model. Section 3 discusses the data. Section 4 estimates loss aversion across countries, presents the results and tries to link it to micro evidence by applying cultural dimensions constructed by Hofstede et al. (2010) and/ or data from the World Values Survey. Section 5 analyses whether and in what manner differences in loss aversion correlate with economic fundamentals. Section 6 then concludes the paper.

\section{The model}

In the macroeconomic model, we assume a non-time-separable utility function, as inspired by Kahneman and Tversky's prospect theory (Kahneman and Tversky, 1979; Tversky and Kahneman, 1992). The subsequent empirical section then tests whether loss aversion can be found in macroeconomic time series. For this aim, we will estimate the Euler equation predicted by the non-standard prospect utility function. To apply GMM when estimating the stochastic Euler equation, we assume a parametric form of loss aversion.

In Kahneman and Tversky's prospect theory, agents value their prospects in terms of gains and losses relative to a reference point. They are loss averse, which means that they are more averse to losses than gain seeking on the other hand. Furthermore, they perform subjective, non-linear probability transformations whereby they allot higher weights to small probabilities and lower weights to high probabilities. Kahneman and Tversky originally propose a value function that is concave in the region of gains and convex for losses. The basic idea on how to capture loss aversion is the fact that the value function must be steeper in the loss region.

The setup of our model follows Rosenblatt-Wisch (2008). While this model is influenced by some long-standing ideas derived from the field of psychology, it does not attempt to implement all aspects of prospect theory. The focus lies on loss aversion and on thinking in differences. The value function is linear for losses and gains, with a kink at the reference point. The agent generates utility out of negative or positive changes in consumption. This piecewise-linear approximation and the replacement of subjective probability weighting by objective probabilities is a widely accepted approach, particularly in regard to analysing markets on an aggregate level (see e.g. Ait-Sahalia and Brandt, 2001; Barberis et al., 2001; Berkelaar et al., 2004). Berkelaar et al. (2004) deliberately abstract from the power function, since it is difficult to disentangle the effects of loss aversion and risk aversion. For the same reason, they do not apply subjective decision weights.

Taking these thoughts into account, one can define a piecewise-linear prospect utility function:

$$
u\left(\Delta c_{t}\right)= \begin{cases}\Delta c_{t} & \text { if } \Delta c_{t} \geq 0, \\ \lambda \Delta c_{t} & \text { if } \Delta c_{t}<0,\end{cases}
$$

where $\Delta c_{t}=c_{t}-c_{t-1}$. The individual cares about consumption differences but weighs losses more heavily, with the parameter $\lambda>1$ capturing loss aversion. Formally, marginal utility is positive everywhere but larger in the loss region: $0<\frac{\partial u\left(\Delta c_{t}\right)}{\partial \Delta c_{t}}<\frac{\partial u\left(-\Delta c_{t}\right)}{\partial\left(-\Delta c_{t}\right)}$ for $\Delta c_{t} \neq 0$.

In every period, the individual realizes a certain level of consumption and correspondingly a level of the capital stock. This consumption level then becomes the new reference point. Hence, the reference point is dynamically updated: The level realized in every period serves as the new reference point. This choice of the reference point is also in line with the dynamic updating scheme of, 
e.g., Barberis et al. (2001). ${ }^{1}$ Benartzi and Thaler (1995) show that the equity premium puzzle with loss averse agents can be explained if these agents monitor the performance of their portfolios every eight months (given a piecewise-linear value function and a loss aversion coefficient of 2.25) or every year (given Tversky and Kahneman's (1992) cumulative prospect theory).

In our analysis, we account for possible sources of psychological influence in the GMM estimations in Section 4 and run our estimations for different reference-updating horizons, namely, a quarterly, half-yearly and annual updating scheme.

How the reference point is updated exactly is an on-going debate (see e.g. Barberis, 2013). Köszegi and Rabin (2006, 2007, 2009) developed expectations-based reference-dependent preferences. In their works, agents' expectations form the reference point. In addition, utility is generated not only out of gains and losses but also through levels in consumption. Andries (2014) and Pagel (2016) recently applied these ideas to asset pricing and Pagel (2017) to a life-cycle consumption model. Gneezy et al. (2017), on the contrary, provide some evidence on the limitation of expectations-based reference dependence. The application of expectations-based reference dependence to a macroeconomic framework like ours would significantly decrease the degrees of freedom, particularly when estimating the parameters across countries. The same applies when we include utility out of consumption levels as an additional part. Furthermore, the length of our macroeconomic time series limits the simultaneous estimation of several parameters. We will come back to this issue in Section 4.

Thus, for simplicity and tractability, we focus on the two main aspects of prospect theory: loss aversion and thinking in differences. Foellmi et al. (2011) show that a utility function defined over these two aspects generates transitional dynamics different from the standard Ramsey-Cass-Koopmans model. Namely, it leads to excess consumption smoothing and can cause the economy to stay in a steady state of low consumption and low capital. They also include utility out of consumption levels, but they show that the different dynamics compared to the standard case only stem from the prospect utility part, namely, loss aversion and thinking in differences.

Thus, given this prospect utility function in equation (1), the social planner ${ }^{2}$ solves

$$
\max _{\Delta c_{t}, k_{t+1}} E \sum_{t=0}^{\infty} \beta^{t} u\left(\Delta c_{t}\right)
$$

subject to the constraint

$$
f\left(k_{t}\right)+(1-\delta) k_{t}=c_{t}+k_{t+1},
$$

where the production function $f\left(k_{t}\right)$ is strictly increasing and concave, and the production shocks $A_{t}$ (introduced later) are assumed to enter into the production function in a multiplicative manner. $\beta$ is the discount factor, and $0<\beta<1$.

$\Delta c_{t}$ can be expressed as

$$
\Delta c_{t}=f\left(k_{t}\right)+(1-\delta) k_{t}-k_{t+1}-f\left(k_{t-1}\right)-(1-\delta) k_{t-1}+k_{t} .
$$

Substituting the constraint into the objective function, the social planner's problem becomes

$$
\max _{k_{t+1}} E \sum_{t=0}^{\infty} \beta^{t} u\left(f\left(k_{t}\right)+(1-\delta) k_{t}-k_{t+1}-f\left(k_{t-1}\right)-(1-\delta) k_{t-1}+k_{t}\right) .
$$

This can be solved under the condition that there is an interior solution to the above problem. Having linear utility, corner solutions could be an issue. However, the social planner approach unites maximization of households and firms. Even though utility is linear with $\lambda>1$, the production function is concave and, hence, the social planner chooses an interior solution.

The stochastic Euler equation has the following form

$$
\frac{\partial u\left(\Delta c_{t}\right)}{\partial \Delta c_{t}}=E_{t}\left\{\begin{array}{l}
\beta \frac{\partial u\left(\Delta c_{t+1}\right)}{\partial \Delta c_{t+1}}\left(\frac{\partial f\left(k_{t+1}\right)}{\partial k_{t+1}}+(1-\delta)+1\right) \\
-\beta^{2} \frac{\partial u\left(\Delta c_{t+2}\right)}{\partial \Delta c_{t+2}}\left(\frac{\partial f\left(k_{t+1}\right)}{\partial k_{t+1}}+(1-\delta)\right)
\end{array}\right\} .
$$

Equation (6) deviates from the standard Euler equation in a stochastic Ramsey-Cass-Koopmans model. Consumption is no longer time-separable since the objective function is now dependent not only on $c_{t}$ and $c_{t+1}$ but also on $c_{t+2}$. Previous decisions about consumption and capital move the reference point, and this influences current and future expected utility. Thus, current marginal utility is compared not only to marginal utility in the next period but also to marginal utility thereafter.

We will estimate the stochastic Euler equation using the Generalized Method of Moments. GMM goes back to Hansen and Singleton (1982), who introduced the concept of testing the implications of stochastic Euler equations directly using that method. One advantage of GMM is that it does not require full specification of the underlying economy. It is an econometric estimation procedure in which it is possible to estimate parameters in dynamic objective functions without explicitly having to solve for the stochastic equilibrium. GMM estimation allows us to derive parameter estimation of the stochastic Euler equation and to test for overidentification. Similarly, Ait-Sahalia and Brandt (2001) derive an asset pricing Euler equation for loss averse investors, which is then used for GMM estimation and Berkelaar et al. (2004) use GMM to estimate loss aversion in the aggregate U.S. stock market.

To apply GMM, the function to be estimated must be continuously differentiable. However, as noted above, the utility function in equation (1) is not differentiable at the reference point. To perform GMM, Rosenblatt-Wisch (2008) therefore assumes a smooth

\footnotetext{
${ }^{1}$ In Barberis et al. (2001), the reference point is also influenced by history, but the idea of a dynamic status quo is incorporated in their approach.

${ }^{2}$ Markets are complete, and agents behave competitively, so the First Fundamental Theorem of Welfare Economics holds.
} 
parametric auxiliary function such that the utility function is also differentiable at the kink. This can be done by setting up the loss aversion coefficient as a switching function. Under the assumption of loss aversion, $\lambda$ in equation (1) should be greater than 1 in the loss area and exactly 1 in the gain area. Its value should switch as close as possible to the reference point. Such a switching function $g$ $(\cdot)$ for the loss aversion coefficient $\lambda$ can be represented by

$$
g(\Delta c)=1+\frac{\lambda-1}{1+e^{\mu \Delta c}}
$$

where $\mu$ represents the speed of switching.

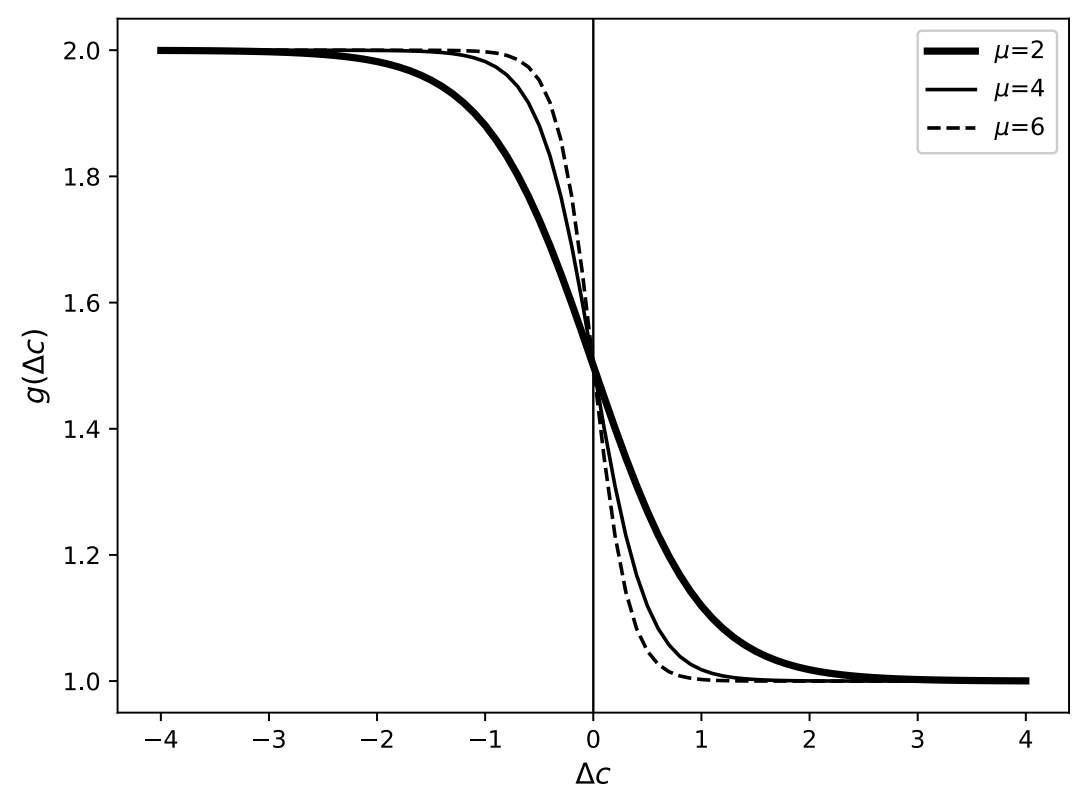

Fig. 1. Switching function. Note: $\mu$ is responsible for the switching speed around the reference point.

The higher $\mu$ is, the faster the switching around zero (see Fig. 1). As required by the assumption of loss aversion, the function $g(\Delta c)$ approaches 1 for $\Delta c>0$ and $\lambda$ for $\Delta c<0$. Thus, expression (7) yields a smooth function to express the loss aversion coefficient $\lambda$ in the model. Inserting (7) for $\lambda$ in the piecewise-linear utility function (1) and denoting the parameterized marginal utility by $\hat{u}^{\prime}(\cdot)$ gives

$$
\hat{u}^{\prime}(\Delta c)=1+\frac{\lambda-1}{1+e^{\mu(\Delta c)}}-\frac{(\lambda-1) \mu \Delta c_{t} e^{\mu \Delta c_{t}}}{\left(1+e^{\mu \Delta c_{t}}\right)^{2}} .
$$

Plugging equation (8) into the Euler equation yields

$$
\begin{aligned}
& 1+\frac{\lambda-1}{1+e^{\mu \Delta c_{t}}}-\frac{(\lambda-1) \mu \Delta c_{t} e^{\mu \Delta c_{t}}}{\left(1+e^{\mu \Delta c_{t}}\right)^{2}}= \\
& E_{t}\left\{\begin{array}{c}
\beta\left(1+\frac{\lambda-1}{1+e^{\mu\left(\Delta c_{t+1}\right)}}-\frac{(\lambda-1) \mu \Delta c_{t+1} e^{\mu \Delta c_{t+1}}}{\left(1+e^{\mu\left(\Delta c_{t+1}\right)}\right)^{2}}\right)\left(\frac{\partial f\left(k_{t+1}\right)}{\partial k_{t+1}}+(1-\delta)+1\right) \\
-\beta^{2}\left(1+\frac{\lambda-1}{1+e^{\mu \Delta c_{t+2}}}-\frac{(\lambda-1) \mu \Delta c_{t+2} e^{\mu \Delta c_{t+2}}}{\left(1+e^{\mu \Delta c_{t+2}}\right)^{2}}\right)\left(\frac{\partial f\left(k_{t+1}\right)}{\partial k_{t+1}}+(1-\delta)\right)
\end{array}\right\} .
\end{aligned}
$$

This is the form we need in order to apply GMM. It can be easily seen that we receive the standard Euler equation of the Ramsey-Cass-Koopmans model when we set $\lambda=1$ in (9). This yields $1=\beta E_{t}\left(\partial f\left(k_{t+1}\right) / \partial k_{t+1}+1-\delta\right)$, which is the first order condition of the corresponding Ramsey-Cass-Koopmans model with linear utility. ${ }^{3}$ Thus, testing for $\lambda=1$ is also an implicit test against/ for the standard Ramsey model.

The production side of the model is specified as follows. The supply side is hit by technological shocks, specified as Solow residuals in the data, which creates the uncertainty in the economy. Output is assumed to be produced with a Cobb-Douglas production function

$$
F\left(A_{t}, K_{t}, L_{t}\right)=Y_{t}=A_{t} K_{t}^{\alpha} L_{t}^{1-\alpha}
$$

\footnotetext{
${ }^{3}$ See also Rosenblatt-Wisch (2005).
} 
and in intensive form, dividing by $L_{t}$, this gives

$$
f\left(k_{t}\right)=y_{t}=A_{t} k_{t}^{\alpha},
$$

where $y_{t}=Y_{t} / L_{t}$ and $k_{t}=K_{t} / L_{t}$. Taking logs and first differences, the Solow residual can then be expressed as

$$
\Delta \ln \left(A_{t}\right)=\Delta \ln \left(y_{t}\right)-\alpha \Delta \ln \left(k_{t}\right),
$$

where $\alpha$ represents the capital share in the production function.

The depreciation rate is set to $\delta=1 .{ }^{4}$ Introducing the Cobb-Douglas type production function into the Euler equation and setting the depreciation rate $\delta=1$ yields:

$$
1+\frac{\lambda-1}{1+e^{\mu \Delta c_{t}}}-\frac{(\lambda-1) \mu \Delta c_{t} e^{\mu \Delta c_{t}}}{\left(1+e^{\mu \Delta c_{t}}\right)^{2}}=E_{t}\left\{\begin{array}{c}
\beta\left(1+\frac{\lambda-1}{1+e^{\mu\left(\Delta c_{t+1}\right)}}-\frac{(\lambda-1) \mu \Delta c_{t+1} e^{\mu \Delta c_{t+1}}}{\left(1+e^{\mu\left(\Delta c_{t+1}\right)}\right)^{2}}\right)\left(\alpha A_{t+1} k_{t+1}^{\alpha-1}+1\right) \\
-\beta^{2}\left(1+\frac{\lambda-1}{1+e^{\mu \Delta c_{t+2}}}-\frac{(\lambda-1) \mu \Delta c_{t+2} e^{\mu \Delta c_{t+2}}}{\left(1+e^{\mu \Delta c_{t+2}}\right)^{2}}\right) \alpha A_{t+1} k_{t+1}^{\alpha-1}
\end{array}\right\} .
$$

Our estimations will be built on this Euler equation. Since we estimate equation (13) using quarterly data, it is worth asking whether a short-run model assuming some combination of nominal and real rigidities might be more appropriate instead. We chose the Ramsey-Cass-Koopmans model because we want to use a most simple benchmark model to analyse the impact of loss aversion. Since we are interested in differences in the degree of loss aversion across countries, a model with nominal rigidities would involve additional behavioural assumptions on the policy functions. As you cannot assume that all countries follow, e.g., the same Taylor rule, we would have to estimate these different parameters even if we assume that all countries follow a monetary rule àla Taylor. With the limited length of time series, the involved loss of the degrees of freedom would reduce the preciseness of the estimates markedly. ${ }^{5}$ Given our focus on cross-country comparisons, the Ramsey-Cass-Koopmans model constitutes a natural starting point and we leave the introduction of further rigidities to future research.

\section{Data}

The following countries are included in our analysis: Austria, Belgium, Bulgaria, Croatia, Cyprus, Czech Republic, Denmark, Estonia, EU, Finland, France, Germany, Greece, Ireland, Italy, Japan, Korea, Latvia, Lithuania, Luxembourg, Malta, Netherlands, Poland, Portugal, Romania, Slovakia, Slovenia, Spain, Sweden, Switzerland, United Kingdom, and the United States. We use quarterly data from 1950 (or the year when they first became available) to 2015, obtained from Datastream. Due to data availability, the sample sizes might differ considerably across countries. However, to make results comparable across countries, we prefer using the same data source, if possible, for all countries, which comes at the price of having fewer data points for some countries. Table A.1 in the appendix lists the countries we included and their abbreviations used in the figures, along with the information regarding which years are covered in the sample.

The data for GDP, consumption and capital stock originate from the OECD, while data for labour are mostly provided by the respective national statistical offices. GDP is measured at constant prices and is seasonally adjusted, as are consumption and the measure for capital stock. Consumption measures private final consumption expenditures, whereas we use gross fixed capital formation to proxy for the capital stock. Labour is measured by total employment, and the exact definitions might differ from country to country. The data for labour are seasonally adjusted as well. GDP and its components are reported in the currency of their respective country, and labour is measured in volumes. We transform GDP, consumption and capital into their intensive form by dividing by labour. The Solow residual is then calculated from a Cobb-Douglas form production function. For each country, data for the capital share $\alpha$ are taken from the Penn World Table, version 9.0 (see Feenstra et al., 2015). To calculate the Solow residual, we use the average capital share over time for each country.

\section{Loss aversion across countries}

\subsection{Estimation: Loss aversion coefficients across countries}

We estimate equation (13) using GMM. An advantage of GMM estimation is that we do not have to know, or to specify, the full economic setting of the underlying economy.

It would be desirable to jointly estimate loss aversion $\lambda$ and the discount factor $\beta$ in equation (13), since these parameters might vary across countries. However, the data at hand are not sufficient to estimate these two parameters jointly. GMM does no longer converge in most specifications when estimating more than one parameter.

For the discount factor $\beta$, we use four different values: $0.90,0.95,0.97$ and 0.99 . We hold the discount factor constant across

\footnotetext{
${ }^{4}$ The depreciation rate enters the calculations of the capital formation stock data (OECD basis) and is as such a part of our physical capital available in the production process.

${ }^{5}$ To get a sense how the higher frequency of data affects our estimates, we redid the estimates using yearly instead of quarterly data. While such a procedure reduces the number of observations leading to less precise estimates, Table A.5 in the appendix shows that our results stay unchanged in qualitative terms. We take this as an indication that our findings are not too sensitive to the frequency of the observations.
} 
Table 1

Results for the US without additional moment conditions.

\begin{tabular}{|c|c|c|c|}
\hline Reference point adj. & 1 quarter & 2 quarters & 4 quarters \\
\hline \multicolumn{4}{|l|}{$\beta=0.90$} \\
\hline$\lambda$ & $1.915^{* * *}$ & $2.464 * * *$ & $4.346^{* * *}$ \\
\hline stv. dev. & 0.128 & 0.302 & 1.138 \\
\hline p-value & 0.000 & 0.000 & 0.003 \\
\hline \multicolumn{4}{|l|}{$\beta=0.95$} \\
\hline$\lambda$ & $1.569 * * *$ & $1.884 * * *$ & $2.960 * * *$ \\
\hline stv. dev. & 0.096 & 0.205 & 0.738 \\
\hline p-value & 0.000 & 0.000 & 0.008 \\
\hline \multicolumn{4}{|l|}{$\beta=0.97$} \\
\hline$\lambda$ & $1.414 * * *$ & $1.633^{* * *}$ & $2.355^{* *}$ \\
\hline stv. dev. & 0.084 & 0.166 & 0.555 \\
\hline p-value & 0.000 & 0.000 & 0.015 \\
\hline \multicolumn{4}{|l|}{$\beta=0.99$} \\
\hline$\lambda$ & $0.825 * * *$ & $1.330 * * *$ & $1.656^{*}$ \\
\hline stv. dev. & 0.043 & 0.125 & 0.351 \\
\hline p-value & 0.000 & 0.008 & 0.062 \\
\hline Nobs & 243 & 243 & 243 \\
\hline
\end{tabular}

Note: $* * * * * *$ denote statistical significance at the $1 \%, 5 \%$ and $10 \%$ level.

Table 2

Summary description of the Hofstede variables.

\begin{tabular}{ll}
\hline Variable & Description \\
\hline Power distance index & $\begin{array}{l}\text { Measures the degree to which less powerful individuals accept that power is distributed unequally. People living in societies } \\
\text { with a high Power Distance accept a hierarchical order in which everyone has his or her place. } \\
\text { Measures the degree of individualism, i.e., to what degree members of a society are only expected to take care of themselves } \\
\text { and their family. People living in societies with a high degree of individualism define their self-image as "I", whereas people in } \\
\text { collectivist societies define themselves as "We". } \\
\text { Measures the importance of achievement and material success in society. Masculine societies tend to be competitive, while } \\
\text { feminine societies are more consensus-oriented. }\end{array}$ \\
Uncertainty avoidance index & $\begin{array}{l}\text { Measures the degree to which the members of society feel uncomfortable with uncertainty or ambiguity. Societies with a } \\
\text { higher score want to try to control the future, while societies with a low score just let the future happen. }\end{array}$ \\
Long term orientation & $\begin{array}{l}\text { Measures how societies value the future in terms of the present and past. Societies that score low view social change with } \\
\text { suspicion, while societies with a high score encourage thrift and education to prepare for the future. } \\
\text { Measures to what degree human drives are regulated by social norms. Indulgent societies allow free gratification of drives } \\
\text { Indulgence vs. restraint }\end{array}$ \\
related to enjoying life and having fun. In restraint societies, gratification is regulated to a stronger degree by strict social \\
norms.
\end{tabular}

Source: Hofstede et al. (2010); Geert Hofstede's website: https://geert-hofstede.com/national-culture.html. More detailed information about the six variables, as well as the measurement of the variables, can be found there.

countries, which is the common approach in current DSGE modelling across countries (see e.g. Justiniano and Preston, 2009). ${ }^{6}$

We take $\alpha$ from the data, as outlined in the previous section. We average $\alpha$ over time for each country and use this value throughout. For the EU, no data for the capital share are available. Therefore, we set $\alpha$ equal to 0.33 , a standard value in the literature. ${ }^{7}$ For computational efficiency, we set $\mu$ equal to 0.1 .

We only report results if we have at least 15 observations, which is true for all countries if we use the full sample. As a special case, we are also investigating whether the loss aversion coefficients across countries have converged over time, with a particular interest in the Euro Area countries after the introduction of the Euro as a single currency. We, therefore, also estimate equation (13) for two sub-samples (pre-2000 and post-2000). However, for the pre-2000 sub-sample, we do not have enough observations for Poland, Czech Republic, Romania, Bulgaria, Malta, Croatia, Cyprus, Lithuania and Greece.

For the specifications of the estimation, we follow the strategy used in Rosenblatt-Wisch (2008). In the baseline specification, we estimate equation (13) without additional moment conditions. As a robustness check, we also introduce additional moment conditions in which we use lagged values as instruments: Assuming individuals form expectations rationally, they use information from

\footnotetext{
${ }^{6}$ Our data only covers well-developed OECD countries with well-integrated financial markets. The discount factor in stochastic models represents a long-run average real return on risky and riskless assets. One could think of a broad portfolio, or from a finance point of view of the market portfolio. With global financial integration this market portfolio can be assessed by each country and should therefore be similar across countries.

${ }^{7}$ See, for example, Abel and Bernanke (2001) or Hall and Taylor (1997). We also performed some robustness checks regarding the capital share. Setting $\alpha=0.33$ for each country does not change our results qualitatively. This finding is robust when using other values for $\alpha$ such as $\alpha$ equal to 0.2 and 0.5 or when using a time-varying $\alpha$ for each country.
} 
Table 3

Selected variables from the World Values Survey.

\begin{tabular}{|c|c|c|}
\hline Variable & Code & Description \\
\hline Work & A030 & Important child qualities: Hard work \\
\hline Timepref & A038 & Important child qualities: Thrift, saving money and things \\
\hline Trust & A165 & Most people can be trusted \\
\hline Optimism & $\mathrm{A} 170$ & Satisfaction with your life \\
\hline Ideas & A189 & Schwartz: It is important to this person to think up new ideas and be creative \\
\hline Status & A190 & Schwartz: It is important to this person to be rich \\
\hline Security & A191 & Schwartz: It is important to this person to live in secure surroundings \\
\hline Altruism & A193 & Schwartz: It is important to this person to help the people nearby \\
\hline Risk & A195 & Schwartz: It is important to this person to be adventurous and to take risks \\
\hline Environment & A197 & Schwartz: It is important to this person to look after the environment \\
\hline Tradition & A198 & Schwartz: It is important to this person to value tradition \\
\hline Genderroles & $\mathrm{C} 001$ & Jobs scarce: Men should have more right to a job than women \\
\hline Freedom & E010 & National goals: Free speech \\
\hline Equality & E035 & Income equality \\
\hline Politics & E039 & Competition: Good or harmful \\
\hline Immigration & E143 & Immigration policy \\
\hline Religion & F050 & Belief in god \\
\hline Fatecontrol & F198 & Fate versus control \\
\hline National & G006 & Pride in nationality \\
\hline
\end{tabular}

period $t$ to form expectations about period $t+1$ but no information from earlier periods. Hence, lagged variables are not correlated with the error terms. In total, we consider seven different specifications concerning the moment conditions. As mentioned, the baseline version is the one without instruments. The other six specifications include lagged values of consumption differences, capital and combinations of it, to formulate additional moment restrictions.

In macroeconomic time series, it is common for the error terms to be correlated over time. Therefore, to allow for heteroscedasticity and autocorrelation in the residuals, we use a heteroscedasticity-and autocorrelation-consistent (HAC) weighing matrix (in case we use instrumental variables) as well as HAC standard errors, using the Bartlett kernel with 4 lags. We use an iterative GMM estimator since it might be more efficient in finite samples (Hall, 2005, p. 88-94), and, as is often the case with macroeconomic time series, our empirical investigation is performed in small samples, which makes this strategy particularly appealing (Hansen et al., 1996).

Furthermore, we verify that all input series are stationary, since GMM relies on the stationarity of the components. The null hypothesis of a unit root (tested by the augmented Dickey-Fuller test) can be rejected for all input series for all countries considered. The consumption series are first-difference stationary. We define the Solow residual in terms of growth rates for technological progress together with the growth rate of capital productivity. Using the exponential of the Solow residual generates a stationary time series for the production part of our Euler equation.

\subsection{Results: Loss aversion coefficients across countries}

First, we confirm the results found in Rosenblatt-Wisch (2008) for a large set of OECD countries. In general, it seems to hold true that we can track loss aversion in an aggregate time series for different countries and across various specifications of the estimated model. Second, and as expected, we find that larger values of $\beta$ lead to lower estimates of the loss aversion parameter. As documented in Rosenblatt-Wisch (2008), a higher value for $\beta$ as well as a higher degree of loss aversion imply that the individual is hurt more by future losses. Hence, $\beta$ and $\lambda$ work in the same direction, which implies that when fixing a data point, the higher $\beta$ is, the lower $\lambda$ has to be and vice versa. This result is confirmed in the data, across specifications as well as across countries.

Table 1 presents the results in detail for one country, namely, the United States. We estimate various specifications with and without instrumental variables. To keep the exposition tractable, some further results are included in the appendix. The estimates are very similar to those found in Rosenblatt-Wisch (2008). Overall, the results reveal highly significant estimates of the loss aversion coefficient.

Tables A.3 and A.4 in the appendix show the results for the United States when using lagged consumption (Table A.3) and lagged capital stock (Table A.4) as an instrument. The results documented in Table 1 can be confirmed. For the specification with $\beta=0.97$, the loss aversion coefficient is estimated to be 1.3 for the semi-annual updating scheme and 2.2 for the annual update scheme, when using lagged consumption as the instrument. These numbers change slightly to 1.6 and 2.3, respectively, when using lagged capital stock as the instrument. All estimates are highly significant. These estimates are close to Tversky and Kahneman's experimentally supported value of 2.25 for the loss aversion coefficient. ${ }^{8}$

These findings carry over to a broad set of OECD countries: Basically, all estimates are above 1, indicating loss aversion and are

\footnotetext{
${ }^{8}$ As mentioned in footnote 5, we also estimated the model for the US with annual data taken from the Penn World Table (version 9.0) as a robustness check for frequency. Table A.5 in the appendix shows the results with an annual updating of the reference point and for the specification without additional moment conditions. The results of the estimates are in line with the ones using quarterly data.
} 
statistically significant. Fig. 2 summarizes the results for the estimates resulting from the specifications without instruments for a discount factor of $\beta=0.97$ and from semi-annual as well as annual reference point updating (for tractability we will use these two specifications as our baseline results for the rest of the paper). We find loss aversion in all countries. The results are somewhat stronger for the semi-annual reference point updating scheme compared to the annual updating scheme.

Furthermore, not only do we find loss aversion in all countries, but we also find cross-country differences in the degree of loss aversion. This holds particularly true for larger updating horizons. Even though the order of the countries when ranked according to their estimated loss aversion coefficient is subject to changes across different specifications, we observe that some country groups are often clustered together at similar loss aversion coefficients.
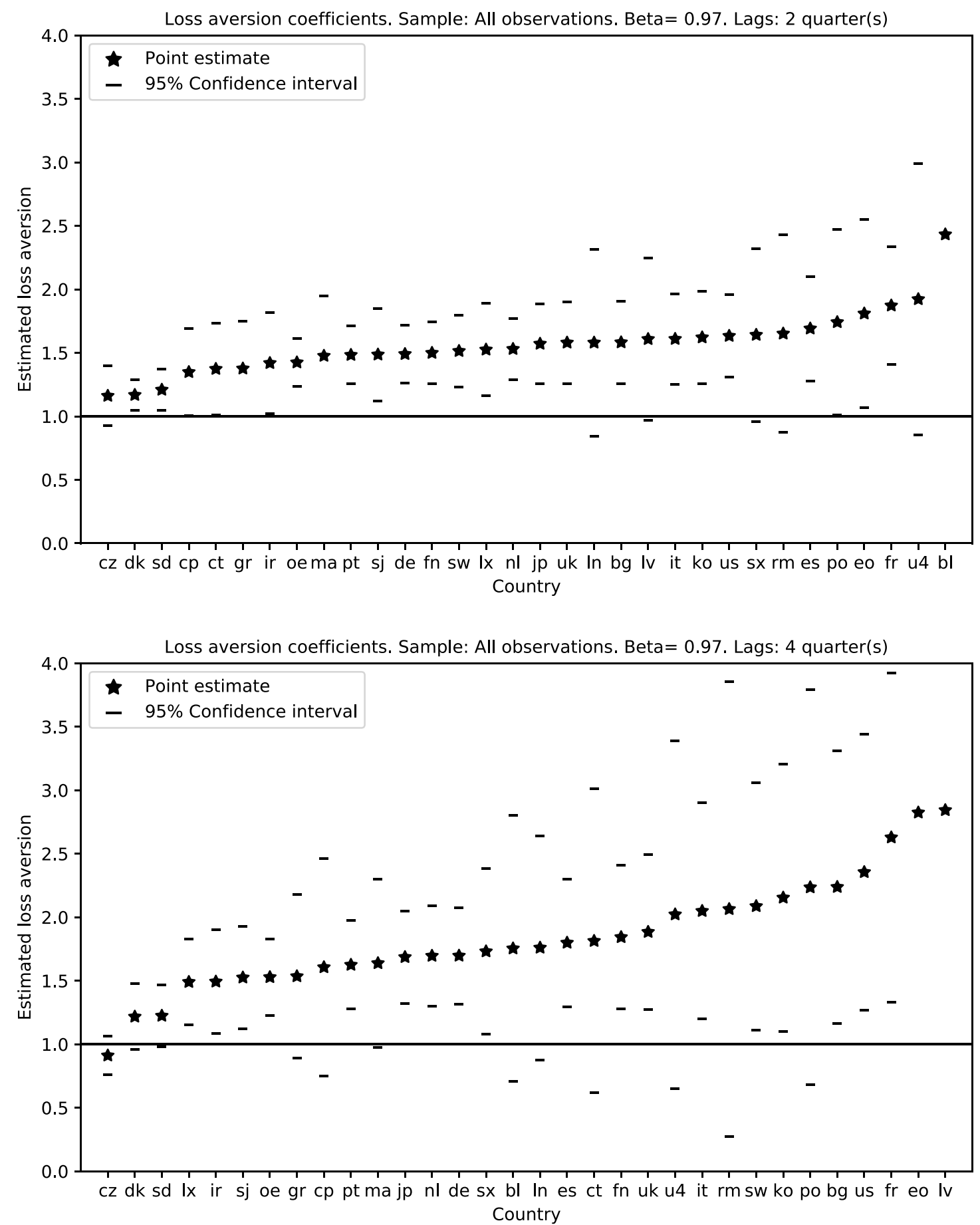

Fig. 2. Estimated loss aversion across countries. Note: Figure in the top (bottom) panel shows results for semi-annual (annual) reference point updating. 
Finally, we test for convergence of loss aversion across countries, comparing the pre-2000 and post-2000 samples. We do not find robust evidence for differences in loss aversion when comparing the pre-2000 sample with the post-2000 sample. Our data do not suggest that we see cross-country convergence in loss aversion. Fig. A.1 in the appendix shows the estimated loss aversion coefficients for the pre-2000 and the post-2000 sample, using $\beta=0.97$ and a semi-annual as well as an annual reference-point updating scheme. Visual inspection does not suggest that the variation in the estimates along the post-2000 axis is smaller than along the pre-2000 axis. To underpin this finding, we report the results from a variance comparison test in Table A.6 in the appendix. There, we test whether the standard deviations of the cross-country estimates are significantly different for the two samples. As the last column reveals, we can reject the null-hypothesis that the standard deviations are the same for only three specifications with an updating horizon of one quarter the specifications in which the standard deviations across countries are very small. For all other specifications, we do not find any evidence that loss aversion has converged.

Conceivably, institutional settings and loss aversion are closely inter-linked. In Table A.7 in the appendix, we repeat the variance comparison test for the sample of countries within the Euro Area only, accounting for the fact that Euro Area countries' preferences could have become more identical after the year 2000, i.e., after having formed a monetary union, or differently said, after having changed the institutional settings. Table A.7, however, shows that convergence in preferences has not taken place to date. We cannot reject the null-hypothesis that the standard deviations of the estimates in the two sub-samples are the same for most specifications.

To sum up, the results found in Rosenblatt-Wisch (2008) for the United States basically carry over to other countries: We consistently find loss aversion coefficients that exceed one (indicating individuals are loss averse), and interestingly, we also find pronounced variation in the size of the loss aversion coefficients across countries.

Can these differences in loss aversion at the aggregate level across countries be matched with micro evidence? We investigate this question in the next subsection. Specifically, we check how our estimated loss aversion coefficients are related to the cultural dimensions reported by Hofstede et al. (2010), as well as how they relate to some key questions from the World Values Survey (WVS).

\subsection{Relation between different degrees of loss aversion and micro evidence}

This subsection analyses how the variation in loss aversion coefficients at the aggregate level is matched with micro evidence.

As our first source of micro evidence, we consider the six cultural dimensions reported by Hofstede et al. (2010) and investigate whether they correlate with our estimated values. This approach follows Wang et al. (2016), who, using data based on surveys, show that loss aversion and the Hofstede dimensions are related. ${ }^{9}$

As our second source of micro evidence, we use data from the World Values Survey to see whether they correlate with our estimated loss aversion coefficients.

To uncover the statistical link between our estimated loss aversion and either the Hofstede cultural dimensions or the values from the WVS, we estimate

$$
L A_{j}=\text { cons }+\gamma \times \text { culture }_{j}+\epsilon_{j}
$$

applying OLS. $L A_{j}$ is the estimated loss aversion coefficient for country $j$, while culture $e_{j}$ is a culture variable from the Hofstede or WVS data.

\subsubsection{Data: Hofstede et al. (2010) and World Values Survey}

The Hofstede et al. (2010) dimensions consist of six variables: Power distance index (PDI), Individualism versus collectivism (IDV), Masculinity versus femininity (MAS), Uncertainty avoidance index (UAI), Long term orientation versus short term normative orientation (LTO) and Indulgence versus restraint (IND). The data result from surveys conducted in several years. However, the data do not have any time dimension; it is a cross-section rather than a panel. Table 2 briefly introduces and describes these variables; more information about the variables can be obtained from Geert Hofstede's website (see source of Table 2).

Descriptive statistics for the Hofstede variables used here are provided in part I of Table A.2 in the appendix. Wang et al. (2016) use only the first four of these dimensions to establish a link between them and loss aversion, mostly on the individual level. They find that individuals with a higher value for PDI and IDV are more loss averse and that individuals living in countries with a higher value for MAS are more loss averse. However, they do not include LTO and IND in their paper.

Our second source, the World Values Survey ${ }^{10}$, includes more than 800 individual questions. Hence, we are required to select some key variables that we consider to have an impact on our estimate of loss aversion. Table 3 lists our selected variables, while we provide descriptive statistics in part II of Table A.2 in the appendix. Variable is how we name them, and Code is the code for the question asked in the WVS data. Description is a short description of the content of the variable. The variables are selected partly because we think they are important for economic outcomes and partly because they were used in earlier economic studies. For example, the question we selected to measure time preferences, A038, was used in Galor and Oezak (2016) to proxy for long-term orientation or patience.

For these variables, we compute the average for each country, i.e., for each country and question pair, we take the simple mean to reduce individual observations to one observation per country, similar to Hofstede et al. (2010)'s calculations of country averages for individual questions that constitute one dimension (see, for example, Hofstede et al., 2010, p. 55). This procedure yields, for each

\footnotetext{
${ }^{9}$ Another reason for differences in loss aversion across countries could be climate, as Galor and Savitskiy (2018) argue.

${ }^{10}$ The WVS data can be obtained from http://www.worldvaluessurvey.org/wvs.jsp.
} 
country, an estimated loss aversion parameter, six Hofstede dimension values and 19 values from the World Values Survey. We then normalize the data on the Hofstede dimensions, as well as the World Values Survey data, by subtracting the minimum of each variable and then dividing by the difference of the maximum and the minimum. Therefore, all values lie between zero and one.

\subsubsection{Relation between loss aversion and culture \& values}

Comparing the six Hofstede dimensions with our estimates of loss aversion, we find that our estimates of loss aversion do not significantly correlate with the four dimensions shown in Wang et al. (2016). Interestingly, however, for our main specifications with $\beta=0.97$, indulgence, one of the dimensions not used by Wang et al. (2016) seems to be significantly negatively correlated with our estimate of loss aversion. Fig. 3 shows this relationship.

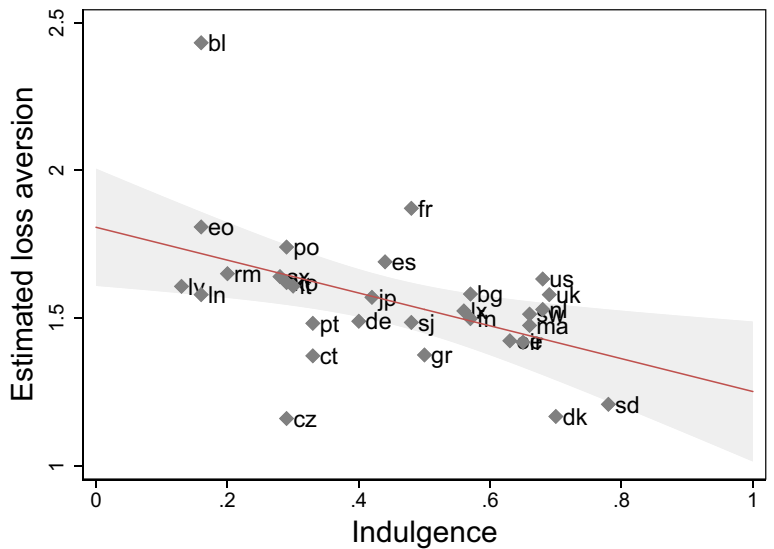

Regression coefficient: -.56 ----- p-value: .01 ----- nObs: 30

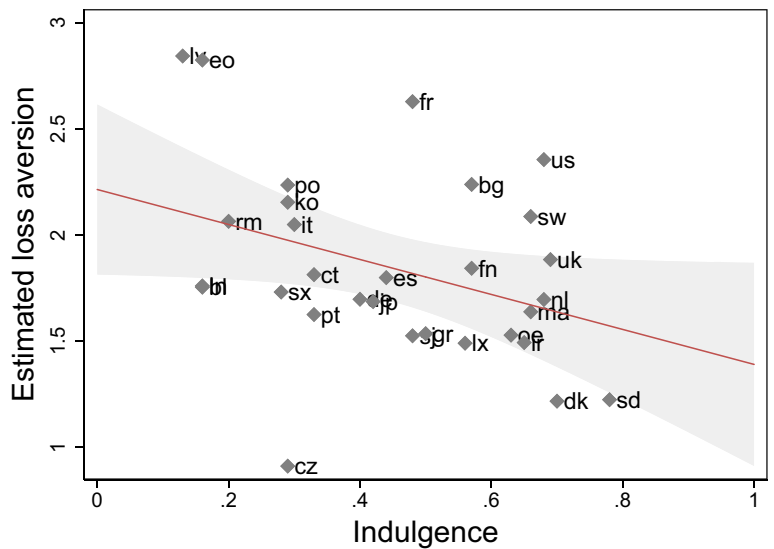

Regression coefficient: -.82 ----- p-value: .05 ----- nObs: 30

Fig. 3. Estimated loss aversion and indulgence. Note: Figure in the left (right) panel shows results for semi-annual (annual) reference point updating.

The left panel in Fig. 3 uses the estimated loss aversion coefficient with a semi-annual updating scheme, whereas the right panel uses the results from the specification with an annual scheme. Indulgence measures how individuals are able to control their impulses. A lower score implies that individuals are more restrained (i.e., more able to control their impulses and desires), which is related to a higher degree of loss aversion. Furthermore, we find that long-term orientation, the last remaining dimension and not shown in Wang et al. (2016), is positively correlated with loss aversion. However, the link is not statistically significant. The results for indulgence and long-term orientation seem to be in line with the status quo bias that loss aversion induces (see Samuelson and Zeckhauser, 1988). The more loss averse an agent is, the higher is his status quo bias. The status quo bias can be interpreted as a longterm orientation and as not being tempted by short-sighted impulses and desires.

For the selected indicators from the World Values Survey, a similar picture emerges: Most of the variables do not seem to be statistically significantly correlated with our estimates of loss aversion. One indicator that seems to have some explanatory power for loss aversion is optimism: Pessimistic people show higher loss aversion. This relationship is shown in Fig. 4.

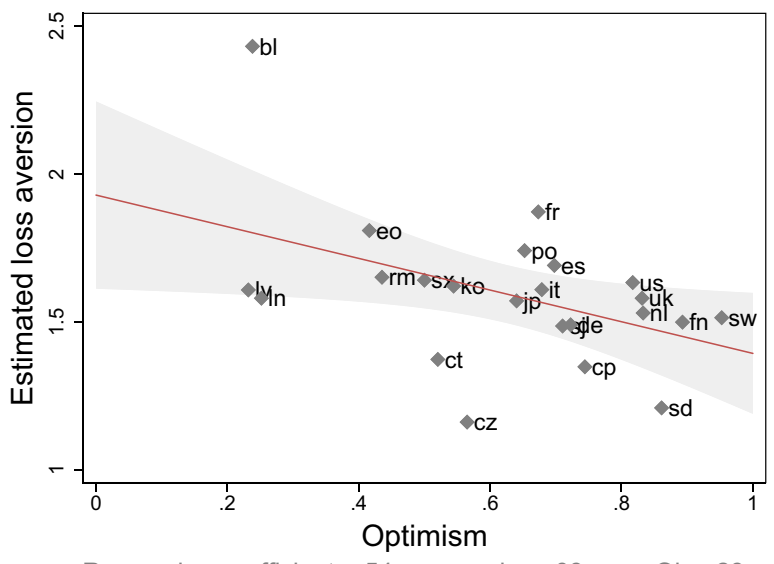

Regression coefficient: -.54 ----- p-value: .03 ----- nObs: 23

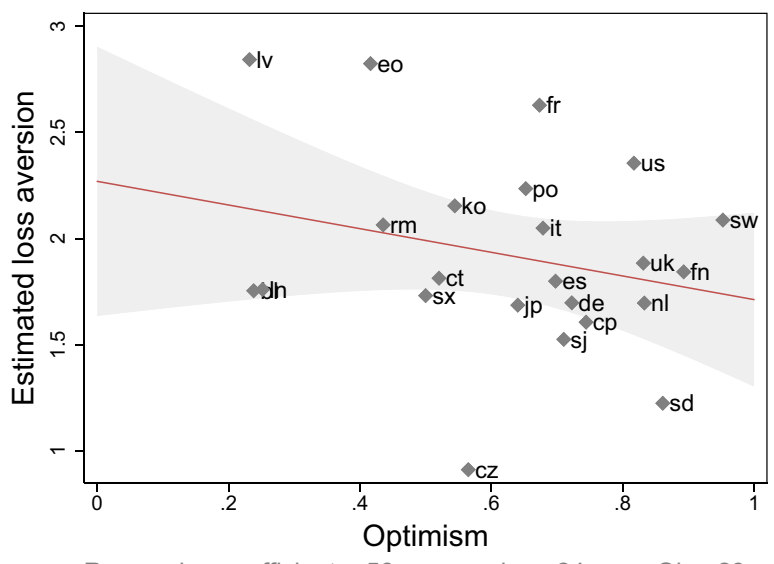

Regression coefficient: -.56 ----- p-value: .24 ----- nObs: 23

Fig. 4. Estimated loss aversion and optimism. Note: Figure in the left (right) panel shows results for semi-annual (annual) reference point updating. 
Again, the result seems intuitively plausible. Taking risks and moving away from the status quo could generate gains but might also generate losses. Pessimistic people would expect a higher likelihood for losses in general, and these losses loom large because of loss aversion. Therefore, pessimistic people would prefer the status quo, and a high status quo bias goes hand in hand with high loss aversion.

However, overall, we find little statistical evidence that either the Hofstede dimensions or the World Values Survey data can be linked to the cross-country variance in the estimated loss aversion coefficients, at the aggregate level. This could be because the power of our statistical tests is limited because we only have a small number of observations. Alternatively, due to large heterogeneity and as noted by, e.g., Becker et al. (2015) or Frey and Gallus (2014), simple aggregation of micro evidence might not be able to successfully gauge preferences at the aggregate level.

\section{Loss aversion and its relation to economic fundamentals}

Previous studies investigating individual preferences suggest that these might influence a country's growth trajectory (Becker et al., 2015). For example, a lower level of patience might reduce a country's savings rate, which in turn will lower its accumulated capital. Foellmi et al. (2011) find that an economy with loss averse agents might be stuck in a steady state with low consumption and low capital because loss averse individuals are reluctant to reduce consumption today in order to achieve a higher steady state tomorrow. Furthermore, they show that the presence of loss aversion leads to stronger consumption smoothing.

Hence, we investigate whether our estimated loss aversion coefficients (again with the specification of $\beta=0.97$ ) are correlated with a series of economic fundamentals series, such as GDP per capita, consumption, savings rates, inflation, investment shares, monetary aggregates and long-term interest rates. Furthermore, we also look at correlations between unemployment benefits and financial openness with loss aversion. Since the estimated loss aversion coefficients are constant over time, we select the economic fundamentals from the year 2010 as well as the year 2000 to exclude potential effects of the crisis. Furthermore, we look at averages over the years as well as fluctuations of these variables over the years, in order to capture long-term trends as well as business cycle fluctuations of these variables.

As we only have 32 observations, we look at bivariate relationships. Obviously, many other factors affect a country's growth trajectory or other economic fundamentals, while driving loss aversion at the same time. However, due to data limitations, this section focuses on correlations only. By doing so, we shed some light on potential links between loss aversion and economic fundamentals, without claiming any causal relationship.

\subsection{Data}

We retrieve data for the economic fundamentals from standard macroeconomic data sources. For the long-term interest rates, we use 10-year government bond yields from the OECD database. For the monetary aggregates, we use the broad money (M3) index taken from the OECD database as well. From the same database, we include data on the replacement ratio (for a single individual having worked full time) and an index of financial services restrictions to proxy financial openness. Real GDP and consumption are taken from the Penn World Table (version 9.0) and adjusted to per-capita terms, using population data from the same database. ${ }^{11}$ Additionally, from the Penn World Table, we take shares of household consumption and government consumption. Finally, we use annual inflation (of consumer prices), broad money (M3) as a \% of GDP and savings rates reported in the World Development Indicators (WDI), provided by the World Bank. Summary statistics for these variables can be found in part III of Table A. 2 in the appendix. For the loss aversion coefficients, we use our point estimates, using the baseline specifications without additional moment restriction, a discount factor of $\beta=0.97$ and semi-annual and annual reference point adjustments.

\subsection{Results}

We investigate the statistical link between the economic fundamentals introduced above and the estimated loss aversion, applying OLS. Hence,

$$
Y_{j}=\text { cons }+\theta \times L A_{j}+v_{j}
$$

where $Y_{j}$ is any economic fundamental in country $j$, either at a given point in time (i.e., in either the year 2000 or 2010 ), or the average over time, or (in the case of consumption smoothing) the standard deviation over time. $L A_{j}$ again is the estimated loss aversion in country $j$.

Among the economic fundamentals investigated, we find a consistent and significant effect for GDP per capita and consumption: Less loss aversion is significantly correlated with higher consumption levels as well as GDP per capita. ${ }^{12}$ Figs. 5 and 6 summarize this result. Here, we use the average of GDP per capita over the same sample for which we have data to estimate the loss aversion

\footnotetext{
${ }^{11}$ We use GDP and consumption data from the Penn World Table here as a standard source for macroeconomic data. Note, that they are only available at annual frequency, which is sufficient for the exercise in this section. For the estimations of the loss aversion parameters, we used quarterly data from the OECD database.

${ }^{12}$ One might object that this correlation could happen by construction since we estimated loss aversion with consumption data. Note however, that this statement refers to levels in consumption, whereas loss aversion is estimated using consumption differences.
} 
coefficient. For Switzerland, for example, we have data from 1970 onward to estimate the Euler equation (see Table A.1 in the appendix), and, hence, we calculate, in this case, the average GDP per capita since 1970. Again, the left panel uses semi-annual reference point updating, whereas the right panel uses annual updating.
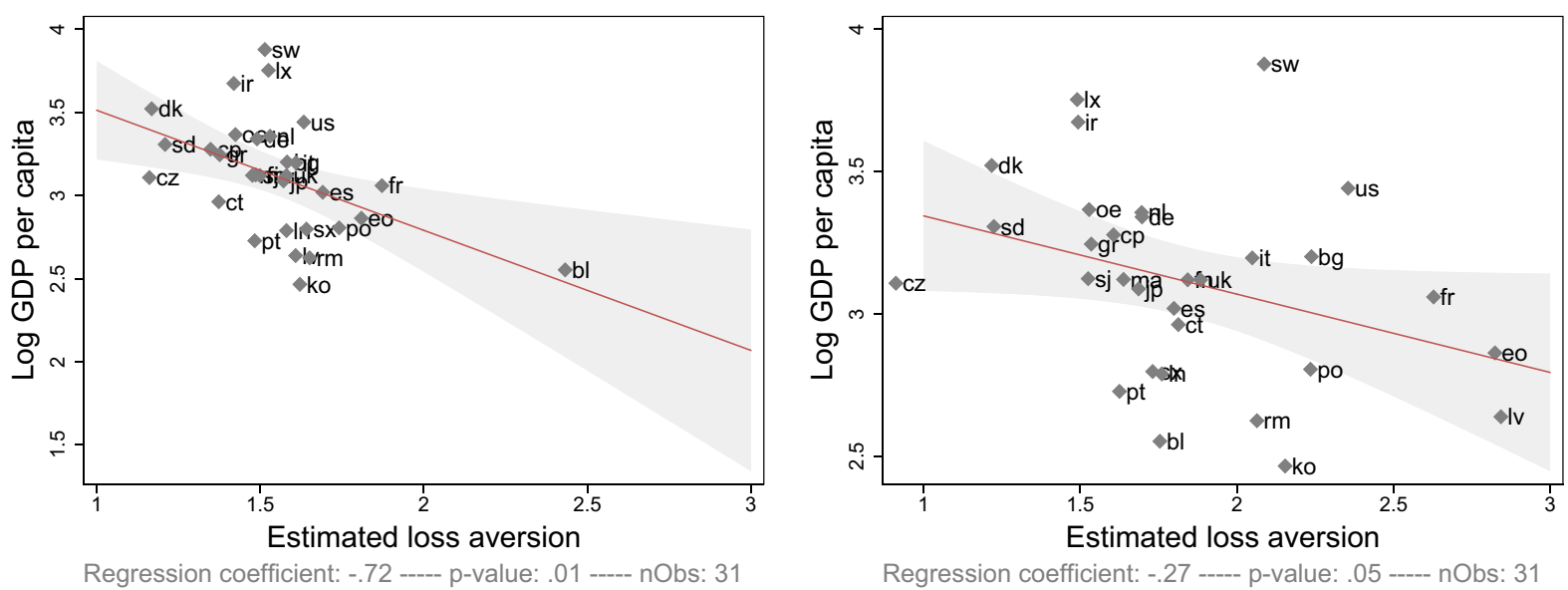

Fig. 5. Estimated loss aversion and average GDP per capita. Note: Figure in the left (right) panel shows results for semi-annual (annual) reference point updating.

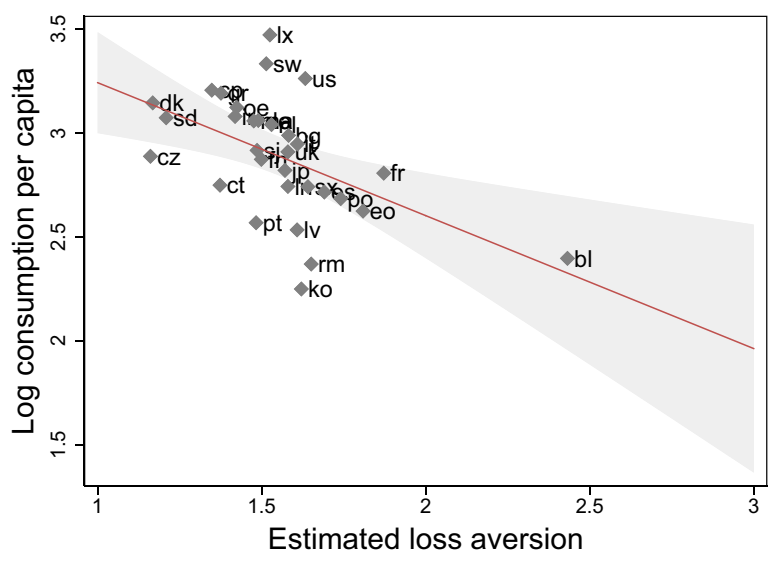

Regression coefficient: -.64 ----- p-value: 0 ----- nObs: 31

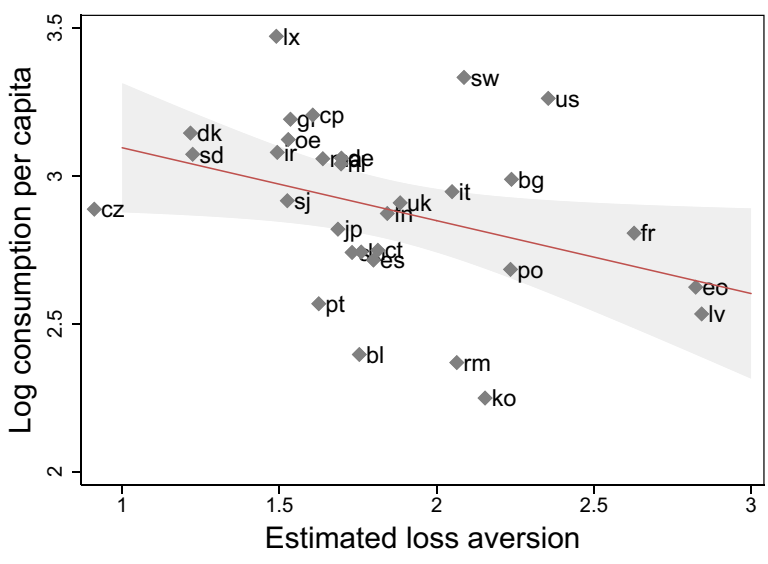

Regression coefficient: -.25 ----- p-value: .04 ----- nObs: 31

Fig. 6. Estimated loss aversion and average consumption per capita. Note: Figure in the left (right) panel shows results for semi-annual (annual) reference point updating.

These results do not change qualitatively when using data of the year 2000 or data of the year 2010, instead of taking the average over time. We find empirical evidence for the theoretical predictions that higher loss aversion relates to lower income and consumption.

Concerning the savings rate, we find a negative correlation between loss aversion and the savings rate, in line with what theory would predict. However, the correlation is statistically insignificant in most specifications.

For inflation, the relationship with loss aversion is positive in all specifications, but the correlation is not significant. The results are similar for the long-term interest rate and the measure of financial regulation (i.e., financial openness is related to lower levels of loss aversion). For the broad money stock M3 and the replacement rate, no patterns can be observed.

What about consumption smoothing? Theory predicts that a higher degree of loss aversion goes hand in hand with more consumption smoothing. Therefore, we calculate the standard deviation of the share of household consumption in output over the years 
for each country in our sample. This gives a simple measure of the fluctuations in consumption shares. We expect a negative correlation between this measure and loss aversion. ${ }^{13}$ Fig. 7 illustrates this finding.
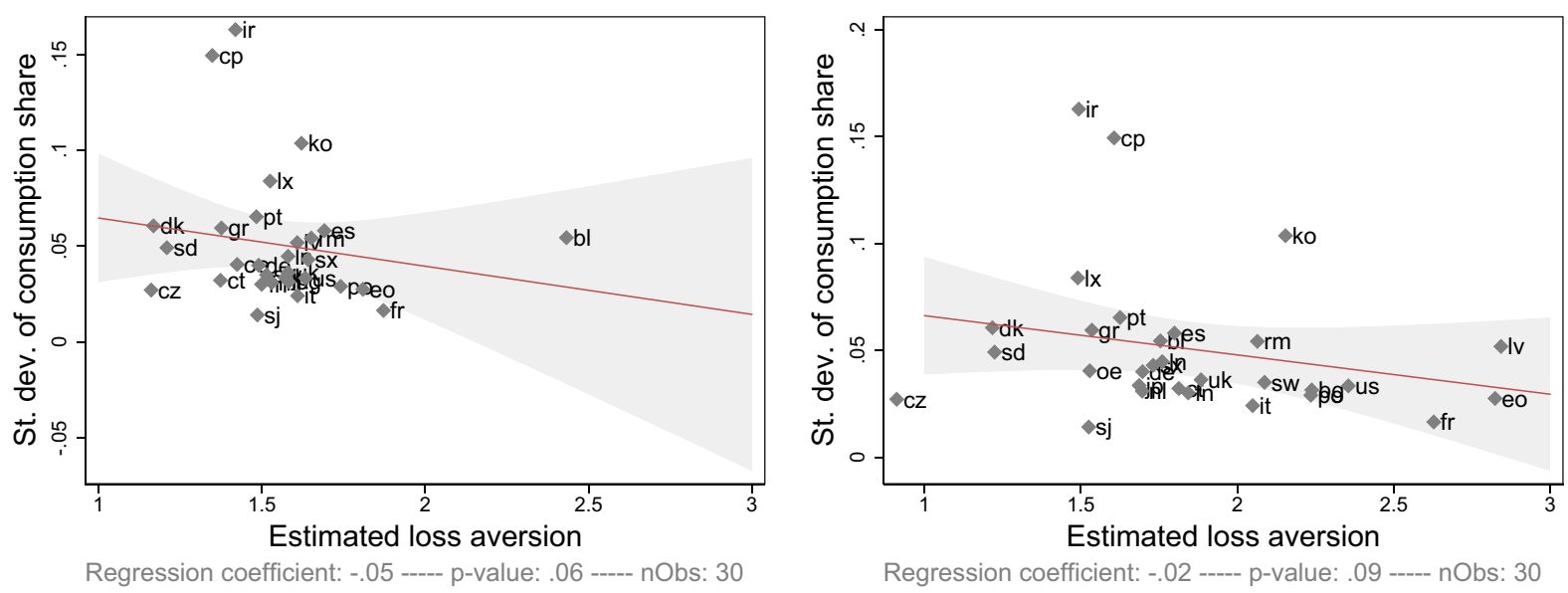

Fig. 7. Estimated loss aversion and fluctuations in consumption. Note: Figure in the left (right) panel shows results for semi-annual (annual) reference point updating.

Looking at the raw correlation, the two measures seem to be negatively correlated, but the relationship is not significant. However, it is likely that a high level of GDP is both negatively correlated with loss aversion and negatively correlated with fluctuations in consumption. Indeed, if we include average GDP over the years in our estimation (by adding average GDP as an additional regressor to equation (15)), the link between the standard deviation of consumption over time and estimated loss aversion becomes statistically stronger. Note that the statistical link found is stronger than suggested by Fig. 7 because we need to control for GDP. As indicated at the bottom of the figures, using semi-annual reference point updating, the p-value is 0.06 , whereas with annual reference point updating, it is 0.09 . Hence, we find some indicative evidence for a link between loss aversion and consumption smoothing, as theory would suggest.

\section{Conclusions}

Preferences of agents matter when thinking about macroeconomic modelling and economic developments. In this paper, we find evidence for loss aversion for a broad set of OECD countries, at the aggregate level. The average degree of loss aversion clearly differs across these countries. To understand these differences, we explore the correlation between loss aversion and macroeconomic fundamentals. We find that GDP per capita and consumption levels are significantly and negatively related to our estimates of loss aversion, in line with what theory would predict. Furthermore, we find a higher degree of consumption smoothing in countries with a higher loss aversion.

To gain more insights on the link between institutions and preferences, we also checked whether loss aversion has converged over time, and, in particular, among Euro Area countries after the introduction of the Euro as the single currency. This seems not to have taken place to date.

To understand the underlying reasons of how reference points are formed, it would be interesting to incorporate expectationsbased reference dependence. However, such an approach would decrease the degrees of freedom substantially, in particular, when estimating the parameters across countries. The data at hand is not sufficient to perform this exercise. However, as time goes by, the length of the macro time series extends. We, therefore, leave this exercise to future research.

\footnotetext{
${ }^{13}$ We exclude Malta here, since its standard deviation of consumption is very large and therefore this data point is a huge outlier.
} 


\section{Appendix A}

\section{A.1. Descriptive statistics}

Table A.1

Countries and sample composition.

\begin{tabular}{|c|c|c|}
\hline Code & Country & First year \\
\hline bg & Belgium & 1960 \\
\hline bl & Bulgaria & 2000 \\
\hline cp & Cyprus & 1999 \\
\hline ct & Croatia & 2002 \\
\hline $\mathrm{cz}$ & Czech Republic & 1994 \\
\hline de & Germany & 1962 \\
\hline $\mathrm{dk}$ & Denmark & 1969 \\
\hline es & Spain & 1961 \\
\hline eo & Estonia & 1995 \\
\hline fn & Finland & 1960 \\
\hline $\mathrm{fr}$ & France & 1950 \\
\hline gr & Greece & 2000 \\
\hline ir & Ireland & 1990 \\
\hline it & Italy & 1960 \\
\hline jp & Japan & 1960 \\
\hline ko & Korea & 1970 \\
\hline $\ln$ & Lithuania & 1998 \\
\hline $\operatorname{lv}$ & Latvia & 1995 \\
\hline lx & Luxembourg & 1985 \\
\hline $\mathrm{ma}$ & Malta & 2000 \\
\hline $\mathrm{nl}$ & Netherlands & 1960 \\
\hline oe & Austria & 1969 \\
\hline po & Poland & 1995 \\
\hline pt & Portugal & 1960 \\
\hline $\mathrm{rm}$ & Romania & 1997 \\
\hline sd & Sweden & 1960 \\
\hline sj & Slovenia & 1995 \\
\hline sw & Switzerland & 1970 \\
\hline sx & Slovakia & 1993 \\
\hline $\mathrm{u} 4$ & EU28 & 1995 \\
\hline $\mathrm{uk}$ & United Kingdom & 1959 \\
\hline us & United States & 1955 \\
\hline
\end{tabular}

Table A.2

Descriptive statistics.

\begin{tabular}{|c|c|c|c|c|c|}
\hline Variable & Mean & Std. dev. & Min. & Max. & $\mathrm{N}$ \\
\hline \multicolumn{6}{|l|}{ Part I: Hofstede variables: } \\
\hline Power distance & 51.03 & 20.14 & 11.00 & 100.00 & 30 \\
\hline Individualism & 57.53 & 19.23 & 18.00 & 91.00 & 30 \\
\hline Masculinity & 47.33 & 24.33 & 5.00 & 100.00 & 30 \\
\hline Uncertainty avoidance & 70.17 & 21.86 & 23.00 & 100.00 & 30 \\
\hline Long term orientation & 59.43 & 19.57 & 24.00 & 100.00 & 30 \\
\hline Indulgence & 44.90 & 19.64 & 13.00 & 78.00 & 30 \\
\hline \multicolumn{6}{|c|}{ Part II: World Values Survey variables: } \\
\hline Trust & 1.68 & 0.14 & 1.36 & 1.89 & 23 \\
\hline Work & 0.53 & 0.26 & 0.10 & 0.90 & 23 \\
\hline Tradition & 2.96 & 0.59 & 2.12 & 4.15 & 17 \\
\hline Immigration & 2.48 & 0.16 & 2.23 & 2.82 & 20 \\
\hline Ideas & 2.87 & 0.37 & 2.09 & 3.63 & 17 \\
\hline Status & 4.48 & 0.31 & 4.07 & 4.98 & 17 \\
\hline Security & 2.73 & 0.41 & 1.96 & 3.51 & 17 \\
\hline Altruism & 2.47 & 0.45 & 1.67 & 3.41 & 16 \\
\hline Risk & 4.05 & 0.37 & 3.41 & 4.84 & 17 \\
\hline Environment & 2.58 & 0.36 & 2.00 & 3.29 & 17 \\
\hline Optimism & 6.66 & 0.93 & 4.90 & 8.11 & 23 \\
\hline Politics & 3.79 & 0.55 & 2.91 & 5.03 & 23 \\
\hline
\end{tabular}


Table A.2 (continued)

\begin{tabular}{|c|c|c|c|c|c|}
\hline Variable & Mean & Std. dev. & Min. & Max. & $\mathrm{N}$ \\
\hline Freedom & 1.71 & 0.24 & 1.35 & 2.05 & 9 \\
\hline Equality & 5.40 & 0.81 & 4.21 & 7.06 & 23 \\
\hline National & 1.80 & 0.25 & 1.37 & 2.22 & 23 \\
\hline Religion & 0.72 & 0.18 & 0.42 & 0.97 & 20 \\
\hline Fatecontrol & 6.61 & 0.49 & 5.59 & 7.44 & 14 \\
\hline Genderroles & 1.93 & 0.09 & 1.76 & 2.18 & 23 \\
\hline Timepref & 0.41 & 0.10 & 0.23 & 0.60 & 23 \\
\hline \multicolumn{6}{|c|}{ Part III: Economic fundamentals variables: } \\
\hline Log GDP per capita & 3.42 & 0.37 & 2.69 & 4.07 & 31 \\
\hline Log consumption per capita & 3.18 & 0.29 & 2.53 & 3.73 & 31 \\
\hline CPI inflation in $\%$ & 1.77 & 1.40 & -1.07 & 6.09 & 32 \\
\hline Share of government consumption & 0.21 & 0.05 & 0.08 & 0.28 & 31 \\
\hline Share of household consumption & 0.57 & 0.08 & 0.40 & 0.75 & 31 \\
\hline $10 \mathrm{y}$ interest rate in $\%$ & 4.08 & 2.03 & 1.15 & 10.34 & 25 \\
\hline Savings rate in $\%$ & 21.34 & 6.64 & 5.58 & 39.35 & 32 \\
\hline Broad money (M3) / GDP & 161.45 & 231.17 & 37.98 & 911.21 & 13 \\
\hline Replacement rate in \% & 58.91 & 14.07 & 29.40 & 86.40 & 29 \\
\hline Financial regulation index $* 100$ & 1.37 & 1.83 & 0.00 & 6.70 & 27 \\
\hline
\end{tabular}

Note: Descriptive statistics include only those countries that are in our sample to estimate the loss aversion coefficient.

The Hofstede variables measure a country's score for each of the six dimensions on a scale from 0 to 100 . As an example, consider the power distance. Societies with a high degree of power distance accept that there is a hierarchical order in which everyone has his or her place. In our sample, the lowest score for power distance is 11 for Austria, indicating that Austrians have strong demands for equalization of power.

The World Value Survey variables we use here are country averages. The values for the variables start at 1 (indicating "disagreement" with the question asked) and go up to a maximum of 10, depending on the question. As an example, consider the trust variable. The statement given to the individuals is "Most people can be trusted", with possible answers "1: Most people can be trusted" and "2: Cannot be too careful".

The economic fundamentals variables reflect some conditions in the countries examined in our sample. For tractability and to keep interpretation simple, we here report the data for the year 2010.

\section{A.2. Additional regression outputs}

Table A.3

Results for the US, using lagged consumption as an instrument.

\begin{tabular}{|c|c|c|c|}
\hline Reference point adj. & 1 quarter & 2 quarters & 4 quarters \\
\hline \multicolumn{4}{|l|}{$\beta=0.90$} \\
\hline$\lambda$ & $1.872^{* * *}$ & $2.013^{* * *}$ & $5.324^{* *}$ \\
\hline stv. dev. & 0.305 & 0.390 & 1.878 \\
\hline p-value & 0.004 & 0.009 & 0.021 \\
\hline \multicolumn{4}{|l|}{$\beta=0.95$} \\
\hline$\lambda$ & $1.518^{* *}$ & $1.464^{* * *}$ & $3.119 * *$ \\
\hline stv. dev. & 0.231 & 0.179 & 1.080 \\
\hline p-value & 0.025 & 0.009 & 0.050 \\
\hline \multicolumn{4}{|l|}{$\beta=0.97$} \\
\hline$\lambda$ & $1.362^{*}$ & $1.268^{* * *}$ & $2.207^{*}$ \\
\hline stv. dev. & 0.196 & 0.103 & 0.638 \\
\hline p-value & 0.065 & 0.009 & 0.058 \\
\hline \multicolumn{4}{|l|}{$\beta=0.99$} \\
\hline$\lambda$ & 0.762 & $1.086^{* * *}$ & $1.357^{*}$ \\
\hline stv. dev. & 0.159 & 0.033 & 0.183 \\
\hline p-value & 0.134 & 0.009 & 0.052 \\
\hline Nobs & 243 & 243 & 243 \\
\hline
\end{tabular}

Note: ${ }^{* * *}, * * *$ denote statistical significance at the $1 \%, 5 \%$ and $10 \%$ level. 
Table A.4

Results for the US, using lagged capital as an instrument.

\begin{tabular}{|c|c|c|c|}
\hline Reference point adj. & 1 quarter & 2 quarters & 4 quarters \\
\hline \multicolumn{4}{|l|}{$\beta=0.90$} \\
\hline$\lambda$ & $1.943 * * *$ & $2.459 * * *$ & $4.294 * * *$ \\
\hline stv. dev. & 0.131 & 0.302 & 1.125 \\
\hline p-value & 0.000 & 0.000 & 0.003 \\
\hline \multicolumn{4}{|l|}{$\beta=0.95$} \\
\hline$\lambda$ & $1.591 * * *$ & $1.877^{* * *}$ & $2.917 * * *$ \\
\hline stv. dev. & 0.100 & 0.203 & 0.724 \\
\hline p-value & 0.000 & 0.000 & 0.008 \\
\hline \multicolumn{4}{|l|}{$\beta=0.97$} \\
\hline$\lambda$ & $1.434 * * *$ & $1.626^{* * *}$ & $2.319 * *$ \\
\hline stv. dev. & 0.087 & 0.164 & 0.541 \\
\hline p-value & 0.000 & 0.000 & 0.015 \\
\hline \multicolumn{4}{|l|}{$\beta=0.99$} \\
\hline$\lambda$ & $0.835 * * *$ & $1.324 * * *$ & $1.629 *$ \\
\hline stv. dev. & 0.042 & 0.122 & 0.336 \\
\hline p-value & 0.000 & 0.008 & 0.061 \\
\hline Nobs & 241 & 241 & 241 \\
\hline
\end{tabular}

Note: $* * * * * *$ denote statistical significance at the $1 \%, 5 \%$ and $10 \%$ level.

Table A.5

Results for the US without additional moment conditions and using annual data.

\begin{tabular}{ll}
\hline Reference point adj. & 1 year \\
\hline$\beta=0.9$ & \\
$\lambda$ & $2.379^{* * *}$ \\
std. dev. & 0.509 \\
p-value & 0.007 \\
$\beta=0.95$ & \\
$\lambda$ & $1.833^{* *}$ \\
std. dev. & 0.357 \\
p-value & 0.020 \\
$\beta=0.97$ & \\
$\lambda$ & $1.597^{* *}$ \\
std. dev. & 0.296 \\
p-value & 0.044 \\
$\beta=0.99$ & \\
$\lambda$ & 1.312 \\
std. dev. & 0.229 \\
p-value & 0.173 \\
Nobs & 62 \\
\hline
\end{tabular}

Note: ${ }^{*}, * * * * *$ denote statistical significance at the $1 \%$, $5 \%$ and $10 \%$ level. 


\section{A.3. Loss aversion for different sub-samples}

Table A.6

Estimated loss aversion for different sub-samples.

\begin{tabular}{|c|c|c|c|c|c|c|c|}
\hline \multirow[b]{2}{*}{$\beta$} & \multirow[b]{2}{*}{ Lag } & \multirow[b]{2}{*}{ Nobs } & \multicolumn{2}{|l|}{ Mean } & \multicolumn{2}{|l|}{ St. dev. } & \multirow[b]{2}{*}{$\mathrm{p}$-value } \\
\hline & & & Pre-2000 & Post-2000 & Pre-2000 & Post-2000 & \\
\hline 0.90 & 1 & 23 & 1.95 & 1.80 & 0.26 & 0.17 & 0.05 \\
\hline 0.90 & 2 & 23 & 2.47 & 2.26 & 0.59 & 0.49 & 0.41 \\
\hline 0.90 & 4 & 20 & 3.49 & 3.05 & 1.31 & 1.40 & 0.77 \\
\hline 0.95 & 1 & 23 & 1.58 & 1.50 & 0.16 & 0.11 & 0.05 \\
\hline 0.95 & 2 & 23 & 1.88 & 1.77 & 0.37 & 0.30 & 0.35 \\
\hline 0.95 & 4 & 20 & 2.45 & 2.23 & 0.74 & 0.75 & 0.93 \\
\hline 0.97 & 1 & 23 & 1.42 & 1.37 & 0.12 & 0.08 & 0.05 \\
\hline 0.97 & 2 & 23 & 1.62 & 1.56 & 0.28 & 0.22 & 0.30 \\
\hline 0.97 & 4 & 20 & 2.01 & 1.88 & 0.50 & 0.51 & 0.96 \\
\hline 0.99 & 1 & 23 & 1.10 & 1.06 & 0.21 & 0.18 & 0.48 \\
\hline 0.99 & 2 & 23 & 1.32 & 1.26 & 0.17 & 0.19 & 0.71 \\
\hline 0.99 & 4 & 20 & 1.50 & 1.44 & 0.24 & 0.34 & 0.14 \\
\hline
\end{tabular}

Note: The table reports an overview of the point-estimates for the different countries. For example, in the column Mean Pre-2000, the cross-country mean of the estimated loss aversion coefficient for the years prior to 2000 is reported, while St. dev. reports the standard deviation across the crosscountry estimates. p-value reports the p-value from a variance comparison test in which the tested hypothesis is that the standard deviations are not the same.

Extreme outliers have been removed from the sample.

Table A.7

Estimated loss aversion for different sub-samples: Euro Area countries.

\begin{tabular}{|c|c|c|c|c|c|c|c|}
\hline \multirow[b]{2}{*}{$\beta$} & \multirow[b]{2}{*}{ Lag } & \multirow[b]{2}{*}{ Nobs } & \multicolumn{2}{|l|}{ Mean } & \multicolumn{2}{|l|}{ St. dev. } & \multirow[b]{2}{*}{$\mathrm{p}$-value } \\
\hline & & & Pre-2000 & Post-2000 & Pre-2000 & Post-2000 & \\
\hline 0.90 & 1 & 17 & 1.94 & 1.74 & 0.26 & 0.12 & 0.00 \\
\hline 0.90 & 2 & 17 & 2.44 & 2.17 & 0.63 & 0.42 & 0.10 \\
\hline 0.90 & 4 & 15 & 3.41 & 2.99 & 1.49 & 1.53 & 0.93 \\
\hline 0.95 & 1 & 17 & 1.57 & 1.47 & 0.16 & 0.08 & 0.01 \\
\hline 0.95 & 2 & 17 & 1.85 & 1.72 & 0.41 & 0.26 & 0.08 \\
\hline 0.95 & 4 & 15 & 2.40 & 2.21 & 0.84 & 0.82 & 0.94 \\
\hline 0.97 & 1 & 17 & 1.42 & 1.34 & 0.13 & 0.07 & 0.01 \\
\hline 0.97 & 2 & 17 & 1.60 & 1.52 & 0.31 & 0.20 & 0.07 \\
\hline 0.97 & 4 & 15 & 1.97 & 1.87 & 0.57 & 0.55 & 0.93 \\
\hline 0.99 & 1 & 17 & 1.11 & 1.07 & 0.21 & 0.16 & 0.32 \\
\hline 0.99 & 2 & 17 & 1.31 & 1.23 & 0.20 & 0.20 & 0.90 \\
\hline 0.99 & 4 & 15 & 1.48 & 1.43 & 0.27 & 0.38 & 0.21 \\
\hline
\end{tabular}

Note: The table reports an overview of the point-estimates for the different countries. For example, in the column Mean Pre-2000, the cross-country mean of the estimated loss aversion coefficient for the years prior to 2000 is reported, while St. dev. reports the standard deviation across the crosscountry estimates. p-value reports the p-value from a variance comparison test in which the tested hypothesis is that the standard deviations are not the same.

Extreme outliers have been removed from the sample. 

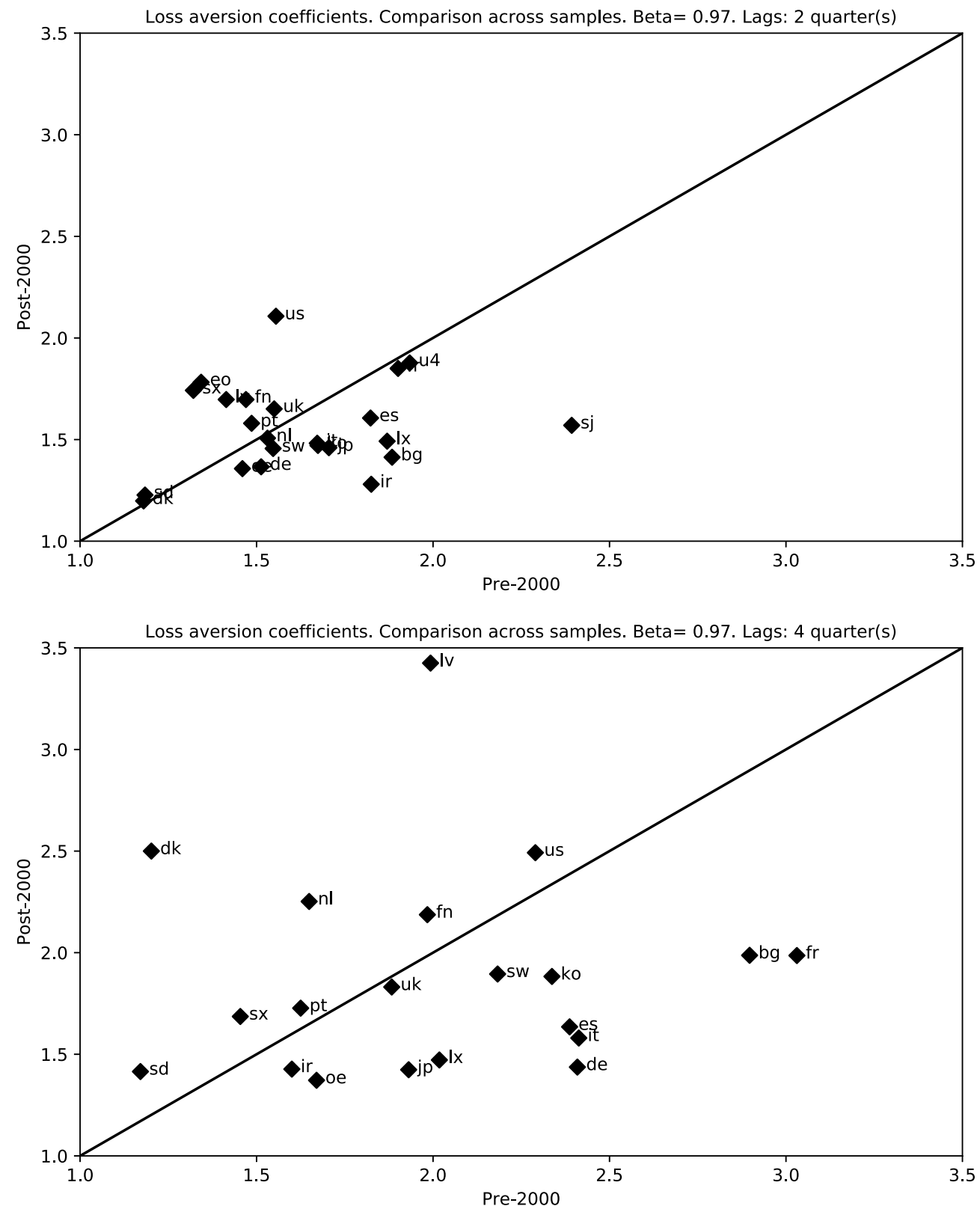

Fig. A.1. Estimated loss aversion before and after 2000. Note: The figure in the top (bottom) panel shows results for semi-annual (annual) reference point updating. The significance classification is taken from the estimation where we use the full sample.

\section{References}

Abdellaoui, M., Bleichrodt, H., Paraschiv, C.P., 2007. Loss aversion under prospect theory: a parameter-free measurement. Manag. Sci. 53 (10), 1659-1674.

Abel, A.B., Bernanke, B.S., 2001. Macroeconomics. Addison Wesley Longman, Boston.

Aït-Sahalia, Y., Brandt, M.W., 2001. Variable selection for portfolio choice. J. Finance 56, 1297-1351.

Andries, M., 2014. Consumption-based asset pricing with loss aversion. Working Paper. Toulouse School of Economics.

Barberis, N., Huang, M., Santos, T., 2001. Prospect theory and asset prices. Q. J. Econ. 116 (1), 1-53.

Barberis, N.C., 2013. Thirty years of prospect theory in economics: a review and assessment. J. Econ. Perspect. 27 (1), $173-196$.

Becker, A., Dohmen, T.J., Enke, B., Falk, A., Huffman, D., Sunde, U., 2015. The nature and predictive power of preferences: global evidence. Technical Report. CEPR Discussion Papers.

Benartzi, S., Thaler, R.H., 1995. Myopic loss aversion and the equity premium puzzle. Q. J. Econ. 110 (1), $73-92$.

Berkelaar, A.B., Kouwenberg, R., Post, T., 2004. Optimal portfolio choice under loss aversion. Rev. Econ. Stat. 86, $973-987$.

Brooks, P., Zank, H., 2005. Loss averse behavior. J. Risk Uncertainty 31, 301-325.

Chen, M.K., Lakshminarayanan, V., Santos, L.R., 2006. How basic are behavioral biases? Evidence from capuchin monkey trading behavior. J. Political Econ. 114 (3), 
$517-537$.

Feenstra, R.C., Inklaar, R., Timmer, M.P., 2015. The next generation of the Penn World Table. Am. Econ. Rev. 105 (10), $3150-3182$.

Foellmi, R., Rosenblatt-Wisch, R., Schenk-Hoppé, K.R., 2011. Consumption paths under prospect utility in an optimal growth model. J. Econ. Dyn. Control 35 (3), 273-281.

Frey, B.S., Gallus, J., 2014. Aggregate effects of behavioral anomalies: a new research area. Econ. Psychol. Choice Theory 8 (18).

Galor, O., Oezak, O., 2016. The agricultural origins of time preference. Am. Econ. Rev. 106 (10), 3064-3103.

Galor, O., Savitskiy, V., 2018. Climatic roots of loss aversion. Working Paper. Brown University.

Gneezy, U., Goette, L., Sprenger, C., Zimmermann, F., 2017. The limits of expectations-based reference dependence. J. Eur. Econ. Assoc. 15 (4), $861-876$.

Hall, A.R., 2005. Generalized Method of Moments. Oxford University Press Oxford.

Hall, R.E., Taylor, J.B., 1997. Macroeconomics. Norton, New York.

Hansen, L.P., Heaton, J., Yaron, A., 1996. Finite-sample properties of some alternative GMM estimators. J. Bus. Econ. Stat. 14 (3), $262-280$.

Hansen, L.P., Singleton, K.J., 1982. Generalized instrumental variables estimation of nonlinear rational expectations models. Econometrica 50 (5), 1269-1286.

Herrmann, B., Thöni, C., Gächter, S., 2008. Antisocial punishment across societies. Science 319 (5868), $1362-1367$.

Hofstede, G., Hofstede, G.J., Minkov, M., 2010. Cultures and Organizations - Software of the Mind. McGraw-Hill Education Ltd.

Justiniano, A., Preston, B., 2009. Monetary policy and uncertainty in an empirical small open economy model. Technical Report. Federal Reserve Bank of Chicago, Working Paper 2009-21.

Kahneman, D., Knetsch, J.L., Thaler, R.H., 1990. Experimental tests of the endowment effect and the coase theorem. J. Political Econ. 98, 1325-1348.

Kahneman, D., Tversky, A., 1979. Prospect theory: an analysis of decision under risk. Econometrica 47 (2), $263-291$.

Kőszegi, B., Rabin, M., 2006. A model of reference-dependent preferences. Q. J. Econ. 121, 1133-1165.

Kőszegi, B., Rabin, M., 2007. Reference-dependent risk attitudes. Am. Econ. Rev. 97, 1047-1073.

Kőszegi, B., Rabin, M., 2009. Reference-dependent consumption plans. Am. Econ. Rev. 99 (3), 909-936.

Pagel, M., 2016. Expectations-based reference-dependent preferences and asset pricing. J. Eur. Econ. Assoc. 14 (2)

Pagel, M., 2017. Expectations-based reference-dependent life-cycle consumption. Rev. Econ. Stud. 84, 885-934.

Rieger, M.O., Wang, M., Hens, T., 2015. Risk preferences around the world. Manag. Sci. 61 (3), 637-648.

Rosenblatt-Wisch, R., 2005. Optimal capital accumulation in a stochastic growth model under loss aversion. Technical Report. NCCR FINRISK Working Paper 222,

University of Zurich.

Rosenblatt-Wisch, R., 2008. Loss aversion in aggregate macroeconomic time series. Eur. Econ. Rev. 52 (7), 1140-1159.

Samuelson, W., Zeckhauser, R., 1988. Status quo bias in decision making. J. Risk Uncertainty 1 (1), 7-59.

Thaler, R.H., 1980. Toward a positive theory of consumer choice. J. Econ. Behav. Org. 1, 36-60.

Tversky, A., Kahneman, D., 1991. Loss aversion in riskless choice: a reference-dependent model. Q. J. Econ. 106, 1039-1061.

Tversky, A., Kahneman, D., 1992. Advances in prospect theory: cumulative representation of uncertainty. J. Risk Uncertainty 5 (4), 297-323.

Vieider, F.M., Lefebvre, M., Bouchouicha, R., Chmura, T., Hakimov, R., Krawczyk, M., Martinsson, P., 2015. Common components of risk and uncertainty attitudes across contexts and domains: evidence from 30 countries. J. Eur. Econ. Assoc. 13 (3), 421-452.

Wang, M., Rieger, M.O., Hens, T., 2016. The impact of culture on loss aversion. J. Behav. Decis. Making 30 (2), $270-281$. 